\title{
$\underline{\mathbf{P}-53}$
}

\section{An Evaluation of Pereskia Bleo Extracts Against $\alpha$-Glucosidase Inhibition}

\author{
Musthahimah Muhamad, Farahdina Man and Choo Chee Yan
}

MedChem Herbal Research Group, Faculty of Pharmacy, Universiti Teknologi MARA,42300 Puncak Alam, Selangor, Malaysia;E-mail:choo715@puncakalam.uitm.edu.my

$\alpha$-Glucosidase is an enzyme catalyzing the breakdown of carbohydrate into glucose in the small intestine. Inhibition of $\alpha$ glucosidase enzyme is considered one of the alternative treatment for type 2 diabetes by reducing postprandial hyperglycemia. Extracts from Pereskia bleo were evaluated on it's $\alpha$-glucosidase inhibition. The ground dried leaves of P.bleo were macerated in methanol at room temperature. The crude methanolic extract was filtered and dried under reduced pressure. The dried crude methanolic extract was resuspended in deionised water and sequentially partitioned with $n$-hexane, chloroform and butanol. The partitioned extracts were dried with a rotary evaporator The inhibitory effects of the four extracts were evaluated for its $\alpha$ glucosidase inhibition using the enzymatic method. Acarbose was used as the positive drug control. At $0.5 \mathrm{mg} / \mathrm{ml}$, the chloroform extract exhibited the highest inhibiton of $69 \%$.

Keyword: $\alpha$-glucosidase, glucose, Pereskia bleo, inhibitory activity. 